\title{
Cofinitely Generalized Lifting Module
}

\author{
Wasan Khalid ${ }^{1}$ and Adnan S. Wadi ${ }^{2 *}$ \\ Department of Mathematics, College of Science, Baghdad University, Baghdad-Iraq \\ *Corresponding Author: adnanwadi76@gmail.com
}

\begin{abstract}
Let $\mathrm{R}$ be an associative ring with identity, and let $\mathrm{M}$ be a unitary left $\mathrm{R}$-module. The main purpose of this paper is to introduce and study properties of class of modules including lifting module. We use the Radical submodule to introduce this class of modules. We start by defining a generalized lifting module as a generalization of lifting module, then we give another generalization of generalized lifting module we called it cofinitely generalized lifting module. Thus, we give characterization and prove some properties of this type of modules. [DOI: 10.22401/ANJS.00.2.12]
\end{abstract}

Keywords: lifting module, Rad- lifting module, cofinitely generalized lifting module.

\section{Introduction}

Let $\mathrm{R}$ be an associative ring with identity, and let $\mathrm{M}$ be a unitary left $\mathrm{R}$-module. It is well known that a submodule $\mathrm{N}$ of an $\mathrm{R}$ module $\mathrm{M}$ is said to be small in $\mathrm{M}$ notationally, $\mathrm{N}<<\mathrm{M}$, if $\mathrm{N}+\mathrm{L}=\mathrm{M}$ for every submodule $\mathrm{L}$ of $\mathrm{M}$ implies $\mathrm{L}=\mathrm{M}$ [1].

It is known that $\operatorname{Rad}(\mathrm{M})$ is the intersection of all maximal submodules of $\mathrm{M}$ equivalently $\operatorname{Rad}(M)$ is the sum of all small submodules of $M$ [1]. A module $M$ is said to be lifting or $\left(\mathbf{D}_{\mathbf{1}}\right)$, if for every submodule $\mathrm{N}$ of $\mathrm{M}$ there exists a direct summand $\mathrm{K} \leq \mathrm{N}$ such that $\frac{N}{K}<<\frac{M}{K}$. Lifting modules have been studied by several authors among them, K. Oshiro [2], Wisbauer [1], P. F. Smith [3] and D. Keskin [4].

In this work we introduce generalized lifting module, cofinitely generalized lifting module. We use the concept of $\operatorname{Rad}(\mathrm{M})$ to introduce a generalized lifting module as a generalization of lifting module we prove some results of this type of modules.

Also we introduce cofinitley generalized lifting module as a generalization of generalized lifting module. We need to add certain conditions to prove some properties of this type of modules. Among these results we get that if $\mathrm{M}=\mathrm{M}_{1} \oplus \mathrm{M}_{2}, \mathrm{M}_{2}$ is finitely generated and $\mathrm{M}$ is a generalized cofinitely lifting, then $M_{1}$ is generalized cofinitley lifting.

\section{Lifting and Radical lifting modules}

In this section we study Rad-lifting module as a generalization of lifting module, and some of the properties of this type of modules are displayed.

\section{Definition 2.1:}

Let $\mathrm{M}$ be an R-module. $\mathrm{M}$ is said to be Rad-lifting if for every submodule $\mathrm{N}$ of $\mathrm{M}$ there exists a direct summand $\mathrm{K}$ of $\mathrm{M}, \mathrm{K} \leq \mathrm{N}$ such that $\mathrm{M}=\mathrm{K} \oplus \mathrm{K}^{\prime}, \mathrm{K}^{\prime} \leq \mathrm{M}$ and $\mathrm{N} \cap \mathrm{K}^{\prime} \leq$ $\operatorname{Rad}(\mathrm{M})$

It is clear that every lifting module is Rad-lifting, but the converse is not true in general. Q as Z-module is Rad-lifting but not lifting. The following theorem gives a characterization of Rad-lifting modules.

\section{Theorem 2.2:}

For any R-module $\mathrm{M}$, the following statements are equivalent:

1. $\quad \mathrm{M}$ is a Rad-lifting module.

2. Every submodule $\mathrm{N}$ of $\mathrm{M}$ can be written as $\mathrm{N}=\mathrm{A} \oplus \mathrm{S}$, where $\mathrm{A}$ is a direct summand of $\mathrm{M}$ and $\mathrm{S} \leq \operatorname{Rad}(\mathrm{M})$.

Proof $(1 \rightarrow 2)$ :

Let $N \leq M$, Since $M$ is a Rad - lifting then there exists a direct summand $\mathrm{K}$ of $\mathrm{M}$, $\mathrm{K} \leq \mathrm{N}$, hence $\mathrm{M}=\mathrm{K} \oplus \mathrm{K}^{\prime}, \mathrm{K}^{\prime} \leq \mathrm{M}$ and $\mathrm{N} \cap \mathrm{K}^{\prime} \leq \operatorname{Rad}(\mathrm{M})$. thus $\mathrm{N}=\mathrm{K} \oplus \mathrm{K}^{\prime} \cap \mathrm{N}$, take $\mathrm{A}=\mathrm{N}$ and $\mathrm{N} \cap \mathrm{K}^{\prime}=\mathrm{S} \leq \operatorname{Rad}(\mathrm{M})$.

Proof $(2 \rightarrow 1)$ :

Let $\mathrm{N} \leq \mathrm{M}$, then By (2), $\mathrm{N}=\mathrm{A} \oplus \mathrm{S}$ where $A$ is a direct summand of $M$ and $S \leq \operatorname{Rad}(M)$, hence $\mathrm{M}=\mathrm{A} \oplus \mathrm{B}, \mathrm{B} \leq \mathrm{M}$, hence $\mathrm{A} \leq \mathrm{N}$ and $\mathrm{B} \cap \mathrm{N}=\mathrm{B} \cap \mathrm{A} \oplus \mathrm{B} \cap \mathrm{S}=\mathrm{B} \cap \mathrm{S} \leq \mathrm{S} \leq \operatorname{Rad}(\mathrm{M})$.

The submodule of Rad-lifting need not be Rad-lifting, Z as Z-module of Q as Z- module 
is not Rad-lifting. However we have the following.

\section{Proposition 2.3:}

Let $M$ be a Rad-lifting module, then every direct summand of $M$ is Rad-lifting module.

\section{Proof:}

Let $\mathrm{N} \leq \mathrm{M}$ such that $\mathrm{N} \leq \oplus \mathrm{M}$, then there exists a direct summand $\mathrm{K}$ of $\mathrm{M}$ such that $\mathrm{M}=\mathrm{N} \oplus \mathrm{K}$ and let $\mathrm{L} \leq \mathrm{N}$ then $\mathrm{L} \leq \mathrm{M}$, since $\mathrm{M}$ is Rad-lifting, then there exists $\mathrm{L}^{\prime} \leq \mathrm{L}$ such that $\mathrm{M}=\mathrm{L}^{\prime} \oplus \mathrm{L}^{\prime \prime}, \mathrm{L}^{\prime \prime} \leq \mathrm{M}$, and $\mathrm{L}^{\prime \prime} \cap \mathrm{L} \leq \mathrm{Rad}$ $(\mathrm{M})$, hence $\mathrm{N} \cap \mathrm{M}=\mathrm{N} \cap \mathrm{L}^{\prime} \oplus \mathrm{N} \cap \mathrm{L}^{\prime \prime}, \mathrm{N}=\mathrm{L}^{\prime} \oplus$ $\mathrm{N} \cap \mathrm{L}^{\prime \prime}$, and $\mathrm{N} \cap \mathrm{L}^{\prime \prime} \cap \mathrm{L} \leq \mathrm{N} \cap \operatorname{Rad}(\mathrm{M})=$ $\operatorname{Rad}(\mathrm{N})$ by [5].

\section{Proposition 2.4:}

Let $\mathbf{M}=\mathrm{M}_{1} \oplus \mathrm{M}_{2}$, be a Rad-lifting module, then $\mathrm{M}_{1}$ and $\mathrm{M}_{2}$ are Rad-lifting module.

\section{Proof:}

Let $N_{1} \leq M_{1}$, then $N_{1} \leq M$ Since $M$ is a Rad-lifting module, then by theorem [2.2 ], $\mathrm{N}_{1}=\mathrm{A} \oplus \mathrm{S}$, where $\mathrm{A}$ is a direct summand of $\mathrm{M}$ and $\mathrm{S} \leq \operatorname{Rad}(\mathrm{M})$. Now $\mathrm{M}=\mathrm{A} \oplus \mathrm{B}, \mathrm{B} \leq \mathrm{M}$, hence $\mathrm{M}_{1}=\mathrm{M}_{1} \cap \mathrm{M}=\mathrm{A} \cap \mathrm{M}_{1} \oplus \mathrm{B} \cap \mathrm{M}_{1}$, then $\mathrm{A} \cap \mathrm{M}_{1} \leq_{\oplus} \mathrm{M}_{1}$, and $\mathrm{M}_{1} \cap \mathrm{S} \leq \mathrm{M}_{1} \cap \mathrm{Rad}$ $(M)=\operatorname{Rad}(M)$ by [5].Similarly for $M_{2}$. Recall that a submodule $\mathrm{N}$ of $\mathrm{M}$ is called fully invariant if $\mathrm{f}(\mathrm{N}) \leq \mathrm{N}$ for every $\mathrm{f} \in$ End $(\mathrm{M})$. [10], and an R-module $\mathrm{M}$ is called a duo module if every submodule of $\mathrm{M}$ is fully invariant [10].

\section{Proposition 2.5:}

Let $\mathrm{M}=\mathrm{M}_{1} \oplus \mathrm{M}_{2}$, be a duo module if $\mathrm{M}_{1}$ and $\mathbf{M}_{2}$ are Rad-lifting module, then $\mathrm{M}$ is Rad-lifting module.

\section{Proof:}

Let $\mathrm{N} \leq \mathrm{M}$, then $\mathrm{N}=\mathrm{N} \cap \mathrm{M}_{1} \oplus \mathrm{N} \cap \mathrm{M}_{2}$, by (proposition 2.1.10) $\mathrm{N} \cap \mathrm{M}_{1} \leq \mathrm{M}_{1}, \mathrm{~N} \cap \mathrm{M}_{2}$ $\leq \mathrm{M}_{2}$. Since $\mathrm{M}_{1}$ and $\mathrm{M}_{2}$ are Rad-lifting modules, then $\mathrm{N} \cap \mathrm{M}_{1}=\mathrm{A}_{1} \oplus \mathrm{S}_{1}, \mathrm{~A}_{1} \leq{ }_{\oplus} \mathrm{M}_{1}$, $\mathrm{M}_{1}=\mathrm{A}_{1} \oplus \mathrm{B}_{1}$ and $\mathrm{S}_{1} \leq \operatorname{Rad}\left(\mathrm{M}_{1}\right)$, and $\mathrm{N} \cap \mathrm{M}_{2}$ $=\mathrm{A}_{2} \oplus \mathrm{S}_{\mathbf{2}}, \mathrm{A}_{2} \leq \oplus \mathrm{M}_{\mathbf{2}}, \mathrm{M}_{2}=\mathrm{A}_{2} \oplus \mathrm{B}_{2}$ and $\mathrm{S}_{2} \leq$ $\operatorname{Rad}\left(\mathrm{M}_{2}\right)$. Now $\mathrm{M}=\mathrm{N} \cap \mathrm{M}_{1} \oplus \mathrm{N} \cap \mathrm{M}_{2}=$ $\left(A_{1} \oplus S_{1}\right) \oplus\left(A_{2} \oplus S_{2}\right)$. Thus $M=\left(A_{1} \oplus A_{2}\right) \oplus$ $\left(\mathrm{S}_{1} \oplus \mathrm{S}_{2}\right)$. Thus $\left(\mathrm{A}_{1} \oplus \mathrm{A}_{2}\right) \leq \mathrm{M}$ and $\left(\mathrm{A}_{1} \oplus \mathrm{A}_{2}\right)$ $\leq_{\oplus} \mathrm{M}$ and $\left(\mathrm{S}_{1} \oplus \mathrm{S}_{2}\right) \leq \operatorname{Rad}\left(\mathrm{M}_{1}\right) \oplus \operatorname{Rad}\left(\mathrm{M}_{2}\right)=$ $\operatorname{Rad}(\mathrm{M})$ therefore $\mathrm{M}$ is Rad-lifting module.

\section{Corollary 2.6:}

Let $\mathrm{M}=\mathrm{M}_{1} \oplus \mathrm{M}_{2} \oplus \ldots \oplus \mathrm{M}_{\mathbf{n}}$ be a duo module. If $\mathrm{M}_{\mathrm{i}}$ is Rad-lifting module for all $\mathrm{i}=1,2, \ldots, \mathrm{n}$, then $\mathrm{M}$ is a Rad-lifting module. Proof: Clearly.

\section{Proposition 2.7:}

Let $\mathrm{M}$ be a Rad-lifting module, let $\mathrm{N}$ be a fully invariant submodule of $\mathrm{M}$, then $\frac{M}{N}$ is Rad-lifting module.

\section{Proof:}

Let $\frac{K}{N} \leq \frac{M}{N}$, then $\mathrm{K} \leq \mathrm{M}$, hence $\mathrm{K}=\mathrm{A} \oplus \mathrm{S}$ where $\mathrm{A}$ is a direct summand of $\mathrm{M}$, i.e., $\mathrm{M}=$ $\mathrm{A} \oplus \mathrm{B}, \mathrm{B} \leq \mathrm{M}$ and $\mathrm{S} \leq \operatorname{Rad}(\mathrm{M})$, thus $\frac{K}{N}=$ $\frac{A \oplus S}{N}=\frac{A+N}{N} \oplus \frac{S+N}{N}$ and $\frac{M}{N}=\frac{A \oplus B}{N}=\frac{A+N}{N} \oplus \frac{B+N}{N}$, hence $\frac{A+N}{N} \leq \oplus \frac{M}{N}$ and $\frac{S+N}{N} \leq \frac{\operatorname{Rad}(\mathrm{M})+\mathrm{N}}{N} \leq$ $\operatorname{Rad}\left(\frac{M}{N}\right)$.

\section{Corollary 2.8:}

The homomorphic image of a duo Radlifting module is Rad-lifting module.

Proof: Clearly By prop.2.7.

\section{Corollary 2.9:}

Let $\mathrm{M}$ be an R-module with $\operatorname{Rad}(\mathrm{M})<<$ $M$. Then $M$ is lifting if and only if $\mathbf{M}$ is Radlifting module.

It is known that every semi-simple module is Rad-lifting module, but the converse in general is not true. For example $\mathrm{Q}$ as $\mathrm{Z}$-module is a Rad-lifting module, but not semi-simple.

The following result gives a certain condition on Rad-lifting module to be semisimple module.

\section{Proposition 2.10:}

Let $\mathrm{M}$ be a Rad-lifting module with Rad $(M)=0$. Then $\mathbf{M}$ is a semi-simple module.

\section{Proof:}

Let $\mathrm{N} \leq \mathrm{M}$. Since $\mathrm{M}$ is Rad-lifting module then there exists a submodule $\mathrm{K} \leq \mathrm{N}$ such that $\mathrm{M} 6 \mathrm{~K} \oplus \mathrm{K}^{\prime}, \mathrm{K}^{\prime} \leq \mathrm{M}$ and $\mathrm{N} \cap \mathrm{K}^{\prime} \leq$ $\operatorname{Rad}(\mathrm{M})=0$, hence $\mathrm{M}=\mathrm{N} \oplus \mathrm{K}^{\prime}$ i.e: $\mathrm{N} \leq_{\oplus} \mathrm{M}$. Then $\mathrm{M}$ is a semi-simple module. 


\section{Cofinitely generalized lifting modules:}

In this section we introduce a cofinitely generalized lifting module as a generalization of Rad-lifting (generalized lifting) modules, some of the properties of this type of modules are discussed.

\section{Definition 3.1:}

Let $\mathrm{M}$ be an $\mathrm{R}$-module. $\mathrm{M}$ is called cofinitely generalized lifting (briefly C. G. lifting) module if for every cofinite submodule $\mathrm{N}$ of $\mathrm{M}$, i.e., $\frac{M}{N}$ is finitely generated there exists a submodule $\mathrm{K}$ of $\mathrm{N}$ such that $\mathrm{M}=\mathrm{K} \oplus \mathrm{K}^{\prime}$ and $\mathrm{N} \cap \mathrm{K}^{\prime} \leq \operatorname{Rad}(\mathrm{M})$.

\section{Theorem 3.2:}

Let $\mathrm{M}$ be an $\mathrm{R}$-module. The following statements are equivalent:

1. $\mathrm{M}$ is C.G.lifting.

2. Every cofinite submodule $\mathrm{N}$ of $\mathrm{M}$ can be written as $\mathrm{N}=\mathrm{A} \oplus \mathrm{S}$, where $\mathrm{A}$ is a direct summand of $\mathrm{M}$ and $\mathrm{S} \leq \operatorname{Rad}(\mathrm{M})$ and $\frac{N}{A}$ cofinite submodule of $\frac{M}{A}$.

3. Every cofinite submodule $\mathrm{N}$ of $\mathrm{M}$, then there exists a direct summand $\mathrm{K}$ of $\mathrm{M}$, such that $\mathrm{K} \leq \mathrm{N}$ and $\frac{\mathrm{N}}{\mathrm{K}} \leq \operatorname{Rad}\left(\frac{\mathrm{M}}{\mathrm{K}}\right)$.

Proof $(1 \rightarrow 2)$ :

Let $\mathrm{M}$ be a C. G. lifting module and let $\mathrm{N}$ be a cofinite submodule of $\mathrm{M}$. Then there exists a submodule $\mathrm{K}$ of $\mathrm{N}$, such that $\mathrm{M}=\mathrm{K}$ $\oplus \mathrm{K}^{\prime}, \mathrm{K}^{\prime} \leq \mathrm{M}$, and $\mathrm{N} \cap \mathrm{K}^{\prime} \leq \operatorname{Rad}(\mathrm{M}), \mathrm{N}=\mathrm{N} \cap$ $\mathrm{M}=\mathrm{N} \cap\left(\mathrm{K} \oplus \mathrm{K}^{\prime}\right)=\mathrm{K} \oplus\left(\mathrm{N} \cap \mathrm{K}^{\prime}\right)$ by(modular law) take $\mathrm{A}=\mathrm{K}$ and $\mathrm{S}=\mathrm{N} \cap \mathrm{K}^{\prime} \leq$ $\operatorname{Rad}(\mathrm{M})$. Now $\frac{\frac{M}{A}}{\frac{N}{A}}=\frac{M}{N}$ but $\frac{M}{N}$ is finite generated, therefore $\frac{\frac{M}{A}}{\frac{N}{A}}$ is finite generated. Thus $\frac{N}{A}$ is cofinite submodule in $\frac{M}{A}$.

Proof $(2 \rightarrow 3)$ :

Let $\mathrm{N}$ be a cofinite submodule of $\mathrm{M}$. Then $\frac{M}{N}$ is finite generated By (2), N $=A \oplus S$ where $\mathrm{A}$ is a direct summand of $\mathrm{M}$ and $\mathrm{S} \leq \operatorname{Rad}(\mathrm{M}) \cdot \frac{\frac{M}{A}}{\frac{N}{A}} \cong \frac{M}{N}$ is finite generated, then $\mathrm{N}$ is cofinite in $\mathrm{M} . \mathrm{A} \leq_{\oplus} \mathrm{M}$, therefore $\mathrm{M}=\mathrm{A}$ $\oplus \mathrm{K}^{\prime}, \mathrm{A} \leq \mathrm{N}$, take $\mathrm{A}=\mathrm{K}$, Now $\mathrm{N} \cap \mathrm{K}^{\prime} . \mathrm{N} \cap$ $\mathrm{M}=\left(\mathrm{A} \oplus \mathrm{K}^{\prime}\right) \cap \mathrm{N}=\mathrm{A} \oplus\left(\mathrm{N} \cap \mathrm{K}^{\prime}\right)$ therefore $\frac{N}{A} \cong \mathrm{N} \cap \mathrm{K}^{\prime}$, we want to show that
$\frac{N}{A} \leq \operatorname{Rad}\left(\frac{M}{N}\right) \cdot \frac{N}{A}=\frac{A \oplus S}{A} \cong \frac{S}{S \cap A} \cong \mathrm{S} \leq \operatorname{Rad}(\mathrm{M}) \leq$ $\operatorname{Rad}\left(\frac{M}{A}\right)$ Thus $\frac{N}{A} \leq \operatorname{Rad}\left(\frac{M}{A}\right)$.

Proof $(3 \rightarrow 1)$ :

Let $\mathrm{N}$ be a cofinite submodule of $\mathrm{M}$. Then there exists a cofinite submodule $\mathrm{K}$ of $\mathrm{M}$, such that $\mathrm{M}=\mathrm{K} \oplus \mathrm{K}^{\prime}, \mathrm{K}^{\prime} \leq \mathrm{M}$, with $\frac{N}{K} \leq \operatorname{Rad}\left(\frac{M}{K}\right)$. To prove $\mathrm{N} \cap \mathrm{K}^{\prime} \leq \operatorname{Rad}(\mathrm{M}) . \mathrm{N}=$ $\mathrm{K} \oplus\left(\mathrm{N} \cap \mathrm{K}^{\prime}\right)$, then $\frac{N}{k} \cong \mathrm{N} \cap \mathrm{K}^{\prime}$, but $\frac{N}{K} \leq \mathrm{Rad}$ $\left(\frac{M}{K}\right)$, therefore $\mathrm{N} \cap \mathrm{K}^{\prime} \leq \mathrm{Rad}(\mathrm{M})$.

\section{Remark and Examples:}

1- Every semi-simple module is C. G. lifting.

2- It is clear that every lifting module is C. G. lifting.

3- Every hollow module is C. G. lifting, but the converse is not true in general. Consider $\mathrm{Q}$ as $\mathrm{Z}$-module, since the only cofinite submodule of $\mathrm{Q}$ is $\mathrm{Q}$, hence $\mathrm{Q}$ is C. G. lifting. but not lifting.

4- Every uniserial module is C. G. lifting module. In particular $\mathrm{Z}_{\mathrm{P}} \infty$ is a C. G. lifting as Z-module. Since every uniserial module is hollow module, and hence it is C. G. lifting.

5- Every local module is C.G. lifting module.

6- If every proper cofinite submodule of $\mathrm{M}$ is small in $M$, Then $M$ is $C$. G. lifting module. Let $\mathrm{N}$ be a cofinite proper submodule of $\mathrm{M}$. Therefore $\mathrm{N} \ll<\mathrm{M}$, then $\mathrm{N} \leq \operatorname{Rad}(\mathrm{M})$.

Then there exists $0 \leq \mathrm{N}$ such that $0 \oplus \mathrm{M}$ $=\mathrm{M} . \mathrm{M} \cap \mathrm{N}=\mathrm{N} \leq \operatorname{Rad}(\mathrm{M})$. Thus every cofinite hollow module is C. G.-lifting.

7- $\mathrm{Z}$ as $\mathrm{Z}$-module is not C. G. lifting module.

8- Let $\mathrm{M}=\mathrm{Z} 8 \oplus \mathrm{Z}_{2}$ be a module. Let $\mathrm{N}=$ $\{(\overline{0}, \overline{0}),(\overline{2}, \overline{1}),(\overline{4}, \overline{0}),(\overline{6}, \overline{1})\}$. The only direct summand of $\mathrm{M}$ contained in $\mathrm{N}$ is $\{(\overline{0}, \overline{0})\}$. If $\mathrm{M}$ is $\mathrm{C}$. $\mathrm{G}$. lifting then $\mathrm{N}=\mathrm{A} \oplus \mathrm{S}$ where $A$ is a direct summand of $M$ and $S \leq$ $\operatorname{Rad}(\mathrm{M})$, if $\mathrm{A}=0$, then $\mathrm{N}=\mathrm{S}$. Therefore $\mathrm{N}$ is not small in $\mathrm{M}$ [since $\mathrm{N}+\mathrm{Z}(\overline{1}, \overline{1})=\mathrm{M}$ ]. Hence $\mathrm{M}$ is not C. G. lifting.

\section{Remark 3.4:}

A submodule of $\mathrm{C}$. G. lifting module is not necessary be C. G. lifting module Q as Zmodule is C. G. lifting, but $\mathrm{Z}$ as $\mathrm{Z}$ - module is not C. G. lifting module. The following gives 
a certain condition on submodule of C. G. lifting module to be C. G. lifting module.

\section{Proposition 3.5:}

Let $\mathrm{M}=\mathrm{M}_{1} \oplus \mathrm{M}_{2}$ be an R-module, where $M_{2}$ is finitely generated and $M$ is C. G. lifting module then $\mathrm{M}_{1}$ is $\mathrm{C}$. G. lifting.

Proof:

Let $\mathrm{M}$ be a C. G. lifting module and let $\mathrm{M}=\mathrm{M}_{1} \oplus \mathrm{M}_{2}$. To prove $\mathrm{M}_{1}$ is $\mathrm{C}$. $\mathrm{G}$. lifting, let $\mathrm{N}$ be a cofinite submodule of $\mathrm{M}_{1}$. Then $\mathrm{N} \leq$ $\mathrm{M}$. Then $\mathrm{N}=\mathrm{A} \oplus \mathrm{S}$ where $\mathrm{A}$ is a cofinite direct summand of $\mathrm{M}$ and $\mathrm{S} \leq \operatorname{Rad}(\mathrm{M})$, therefore $\mathrm{M}=\mathrm{A} \oplus \mathrm{L}, \mathrm{L} \leq \mathrm{M}, \frac{M 1}{N}$ is finitely generated. $\frac{\frac{M}{N}}{\frac{M 1}{N}} \cong \frac{M}{M_{1}} \cong \mathrm{M}_{2}$ is finitely generated and $\frac{M_{1}}{N}$ is finitely generated implies $\frac{M}{N}$ is finitely generated. To prove $\mathrm{A}$ is a direct summand of $M_{1} \cdot M_{1}=M_{1} \cap M=M_{1} \cap(A \oplus L)$ $=A \oplus\left(M_{1} \cap L\right)$. Hence $M_{1}$ is $C$. G. lifting module generated. Implies that $\frac{N}{A}$ is cofinite in $\frac{M_{1}}{N}$.

\section{Corollary 3.6:}

Let $\mathrm{M}$ be a C. G. lifting module and let $\mathrm{N}$ be a cofinite direct summand of $\mathrm{M}$, then $\mathrm{N}$ is C. G. lifting.

Proof:

Clearly By proposition 3.5.

\section{Proposition 3.7:}

Let $M$ be a duo module, if $M=M_{1} \oplus$ $\mathrm{M}_{2}$.and for all $\mathrm{i}=1,2 . \mathrm{M}_{1}$ and $\mathrm{M}_{2}$ are a $\mathrm{C}$. $\mathrm{G}$. lifting module. Then $\mathrm{M}$ is a $\mathrm{C}$. $\mathrm{G}$. lifting module

Proof:

Let $\mathrm{N} \leq \mathrm{M}$ with $\mathrm{N}$ is cofinite in $\mathrm{M}$, and since $\mathrm{M}$ is duo module thus, $\mathrm{N}=\mathrm{N} \cap \mathrm{M}_{1} \oplus \mathrm{N}$ $\cap \mathrm{M}_{2} \cong \frac{M_{1}}{N}+\frac{M 2+N}{N}$ hence $\frac{M 1}{N \cap M 1}$ is finite generated thus, $\mathrm{N} \cap \mathrm{M}_{1} \leq \mathrm{M}_{1}$ and $\mathrm{N} \cap \mathrm{M}_{2} \leq$ $\mathrm{M}_{2}$, then by assumption there exists $\mathrm{K}_{1} \leq \mathrm{N} \cap$ $\mathrm{M}_{1}$ and $\mathrm{K}_{2} \leq \mathrm{N} \cap \mathrm{M}_{2}$ such that $\mathrm{M}_{1}=\mathrm{K}_{1} \oplus \mathrm{L}_{1}$, $\mathrm{L}_{1} \leq \mathrm{M}_{1}$, and $\mathrm{N} \cap \mathrm{M}_{1} \cap \mathrm{L}_{1} \leq \operatorname{Rad}\left(\mathrm{M}_{1}\right)$ and for $\mathrm{M}_{2} \ni \mathrm{K}_{2}, \mathrm{~L}_{2} \in \mathrm{M}_{2}$, such that $\mathrm{M}_{2}=\mathrm{K}_{2} \oplus \mathrm{L}_{2}$, $\mathrm{L}_{2} \leq \mathrm{M}_{2}$, and $\mathrm{N} \cap \mathrm{M}_{2} \cap \mathrm{L}_{2} \leq \operatorname{Rad}\left(\mathrm{M}_{2}\right)$, thus $\mathrm{N}=$ $\mathrm{N} \cap \mathrm{M}_{1} \oplus \mathrm{N} \cap \mathrm{M}_{2}$. Thus $\mathrm{M}=\mathrm{M}_{1} \oplus \mathrm{M}_{2}=\mathrm{K}_{1}+\mathrm{K}_{2}$ $\oplus \mathrm{L}_{1}+\mathrm{L}_{2}, \mathrm{~K}_{1}+\mathrm{K}_{2} \leq \mathrm{N} \cap \mathrm{M}_{1} \oplus \mathrm{N} \cap \mathrm{M}_{2}=\mathrm{N}$ and $\mathrm{N} \cap\left(\mathrm{L}_{1}+\mathrm{L}_{2}\right)=\left(\mathrm{N} \cap \mathrm{M}_{1} \oplus \mathrm{N} \cap \mathrm{M}_{2}\right) \cap\left(\mathrm{L}_{1}+\right.$
$\left.\mathrm{L}_{2}\right)=\left(\mathrm{N} \cap \mathrm{M}_{1}\right) \cap \mathrm{L}_{1} \oplus\left(\mathrm{N} \cap \mathrm{M}_{2}\right) \cap \mathrm{L}_{2} \leq \mathrm{Rad}$ $\left(\mathrm{M}_{1}\right) \oplus \operatorname{Rad}\left(\mathrm{M}_{2}\right)=\operatorname{Rad}(\mathrm{M})$ by [5].

\section{Corollary 3.8:}

Let $\mathrm{M}$ be a duo of an $\mathrm{R}$ - module. If $\mathrm{M}=$ $\mathrm{M}_{1} \oplus \mathrm{M}_{2} \oplus \ldots \oplus \mathrm{M}_{\mathbf{n}}$ and for all $\mathrm{i}=1,2, \ldots, \mathrm{n}$, $M_{i}$ is C. G. lifting. Then $M$ is C. G. lifting module.

Now we will consider the following properties:

$\left(\mathbf{C D}_{2}\right)$ : For any cofinite submodule $\mathrm{N}$ of $\mathrm{M}$, for which $\frac{M}{N}$ isomorphic to a direct summand o f M. Then N is a direct summand of M. Q as $\mathrm{Z}$-module has $\left(\mathbf{C D}_{\mathbf{2}}\right)$ property since $\mathrm{Q}$ is the only cofinite submodule of $\mathrm{Q}$.

$\left(\mathbf{C D}_{3}\right)$ : If $\mathrm{K}_{1}, \mathrm{~K}_{2}$ are cofinite direct summand of $M$ with $M=K_{1}+K_{2}$ then $K_{1} \cap K_{2}$ is also a direct summand of $\mathrm{M}$. It is clear that $\mathrm{Z}_{6}$ as $\mathrm{Z}$ module has $\left(\mathbf{C} \mathbf{D}_{\mathbf{3}}\right)$ property.

\section{Definition 3.9:}

Let $\mathrm{M}$ be an R-module. $\mathrm{M}$ is called cofinitely discrete if it is C. G. lifting and $\mathrm{CD}_{2}$. And $\mathrm{M}$ is called cofinitely quasi-discrete (briefly C. quasi discrete) if $\mathrm{M}$ is C. G. lifting and CD3.

\section{Proposition 3.10:}

Let $\mathrm{M}$ be an $\mathrm{R}$ - module, with $\left(\mathbf{C D}_{\mathbf{2}}\right)$ property. Then $\mathrm{M}$ has $\mathbf{C D}_{\mathbf{3}}$ property.

\section{Proof:}

Let $\mathrm{A}, \mathrm{B}$ be a cofinite direct summand of M. Such that $M=A+B$. To show that $A \cap B$ is a direct summand of $M$. Since $A \leq \oplus M$ and $\mathrm{B} \leq \oplus \mathrm{M}$ then there exist $\mathrm{A}^{\prime}, \mathrm{B}^{\prime}$, such that $\mathrm{M}=$ $\mathrm{A} \oplus \mathrm{A}^{\prime}=\mathrm{B} \oplus \mathrm{B}^{\prime}$.Then $\mathrm{A}^{\prime} \cong \frac{M}{A}=\frac{A+B}{A} \cong$ $\frac{B}{A \cap B} \cong \frac{\mathrm{B} \oplus \mathrm{B}^{\prime}}{A \cap\left(\mathrm{B} \oplus \mathrm{B}^{\prime}\right)}$ (by $2^{\text {nd }}$ isomorphic theorem). To prove That $\mathrm{A} \cap\left(\mathrm{B} \oplus \mathrm{B}^{\prime}\right)$.is cofinite in $\mathrm{M}$. Since $\quad \mathrm{A}^{\prime} \cong \frac{\mathrm{B}^{\prime} \oplus \mathrm{B}}{A \cap\left(B+B^{\prime}\right)} \cong \frac{M}{A}$ is finitely generated,then $\frac{\mathrm{B} \oplus \mathrm{B}^{\prime}}{A \cap\left(B+B^{\prime}\right)}$ is finitely generated,it is implies that $\mathrm{A} \cap\left(\mathrm{B} \oplus \mathrm{B}^{\prime}\right)$ is cofinite submodule in $\mathrm{M}$; but $\mathrm{M}$ has $\mathrm{CD}_{2}, \mathrm{~A} \cap(\mathrm{B} \oplus$ $\left.\mathrm{B}^{\prime}\right) \leq_{\oplus} \mathrm{M}$, but $\mathrm{A} \cap \mathrm{B} \leq \mathrm{A} \cap\left(\mathrm{B} \oplus \mathrm{B}^{\prime}\right) \leq_{\oplus} \mathrm{M}$, therefore $\mathrm{A} \cap \mathrm{B} \leq_{\oplus} \mathrm{M}$. Thus $\mathrm{M}$ has CD3.

\section{Remark 3.11:}

Let $\mathrm{M}$ be an $\mathrm{R}$ - module, then: 
$\mathrm{M}$ is C. discrete $\rightarrow \mathrm{M}$ is C. quasi-discrete $\rightarrow \mathrm{M}$ is C.G.lifting module.

\section{Remark 3.12:}

Let $\mathrm{M}$ be a C. G. lifting module in which every cofinite submodule $\mathrm{N}$ of $\mathrm{M}$ is a direct summand of $\mathrm{M}$. Then $\mathrm{M}$ is $\mathrm{C}$. discrete.

\section{Proof:}

Let $\mathrm{N} \leq \mathrm{M}$.Such that $\frac{M}{N}$ is finitely generated, then there exists a submodule $\mathrm{L}$ of $\mathrm{M}$ such that $\mathrm{M}=\mathrm{N} \oplus \mathrm{L}$. therefore $\frac{M}{N} \cong \mathrm{L}$, hence $\mathrm{M}$ has $\mathrm{CD}_{2}$.

\section{REFERENCES:}

[1] Wisbauer R., Foundations of module and Ring Theory, Gordon and Breach, Philadelphia, 1991.

[2] Oshiro K., Lifting modules, Extending modules and their applications to QFrings, Hokkaido Math. J., 1984, (13), 3310-338.

[3] Keskin D., Smith P. F and Xue W. Rings $\bigoplus$ supplemented, J. Algebra, 1999, 218, 470-487.

[4] Keskin D., Finite direct sum of (D1) modules, Turkish journal of Math., 1998, 22(1), 85-91.

[5] Kasch F., Modules and rings, London New York, 1982,

[6] Clark j., Lomp C., Vanaja N., Wisbauer, Lifting modules. Supplements and projectivity in module theory,, series Frontiers in Mathematics, 2006,

[7] Keskin D., and Xue W., Generalization of lifting module, To appear in Acta Mth. Hungar.

[8] Mohamed, S.H., Müller, B. J., Continuous and discrete modules, London Math. Soc. LNS 147 Cambridge University, 190, Cambridge, 1990.

[9] Abdelkader Ben Laroussi-Hamdouni, On lifting modules, M.Sc. Thesis of Department of Mathematics, College of Science, University of Baghdad, 2001.

[10]Ozcan A.C., Harmanci A., Smith P.F., Duo modules, Glagow Math. J. Trust., 2006, 48, 533-545.

[11]Wang Y. W. D., On Cofinitely lifting modules, Algebra Colloquium, 2010, 17, 659-6663.
[12] Ivanav G., Decomposition of modules over serial rings, Comm. in Algebra, 1975, 3 (11) 1031-1036.AC. 